\title{
COLOR STABILITY ASSESSMENT OF INNOVATIVE TECHNIQUE OF COMPLETE DENTURE FLASKING USING SILICONE MASK TECHNIQUE (CASE-CONTROL STUDY)
}

\author{
Shady M. El-Naggar*, Ahmed Mostafa Esmat** and Ayman F. Elawady**
}

\begin{abstract}
Background: Treatment challenges for completely edentulous patient have traditionally been described as a combination of esthetics, function and comfort. Some studies regarding complete denture esthetics were more important than function or comfort. Denture characterization is modification of the form of the denture base to produce a more natural appearance.
\end{abstract}

Materials and Methods: Acrylic resin complete dentures had been divided into two groups according to flasking technique (group I as control group: conventional three pour flasking technique, group II as test group: silicone mask flasking technique). $\Delta \mathrm{E}$ was calculated for both groups immediately and after seven days of exposure to different staining conditions (Smoke, Coffee and Tea).

Results: Heat cured acrylic resin complete denture processed through silicone mask flasking technique showed statistically significant lower $\Delta \mathrm{E}$ values (as $\mathrm{P}<0.05$ ) than conventionally processed heat cured acrylic resin complete denture.

Conclusion: With the limitations of this study regarding color stability change $(\Delta \mathrm{E})$, depending on flasking technique; the conventional three pour flasking technique showed a higher amount of color change (lower color stability) than the silicone mask flasking technique regarding smoking, tea and coffee coloring agents.

KEY WORDS: Flasking, Silicone Mask, Smoke, Denture, Color Stability, Tea, Coffee.

\section{INTRODUCTION}

The academy of prosthodontics term listed the term (esthetics) as the branch of philosophy with beauty especially with color and form involved in the appearance of a given restoration. Some studies regarding success of complete denture failed to show esthetics as more important than function or comfort. On contrary, Carlsson et al found that esthetics was the most important factor of complete denture success ${ }^{(1)}$.

Dentures can enhance patient's self-image. One of the most important factors affecting attitude

* Lecturer of Removable Maxillofacial Prosthodontics, South Valley University.

** Researcher at Fixed and Removable Prosthodontics Department, National Research Center. 
toward general esthetics in population is facial appearance. Removable complete prosthodontics are more difficult in esthetics wise, as the edentulous state is not considered as baseline ${ }^{(2)}$.

Clinical esthetics and patient needs were the most important step in denture characterization. Setting teeth on wax rims, tried in the patient's mouth, occlusion and appearance were verified. Professionals who passes out of graduate dental schools were expected to be trained in the theory and the practice of teeth setting. Whether it is conventional removable or implant retained complete denture, the principles of esthetics remain the same ${ }^{(3)}$.

Martone stated "the key to esthetics lies in the asymmetry". The creator itself has incorporated some asymmetry even in case of human beings which gives it an individualized appearance. Characterization helps the clinician to incorporate his artistic skills along with the theoretical knowledge of denture fabrication ${ }^{(1)}$.

The smooth, pink and polished surface of an average acrylic denture showed false positive esthetic value. Pound in 1951 incorporated the racial and individual gingival color changes of complete denture through tinting acrylic resin by pigmentation $^{(1)}$.

Restoring normal colors and natural contour of gingiva is very critical step to improve esthetics as described as pink esthetics. This can be achieved by proper choose of acrylic resin material, scalloping, stippling and bony prominences ${ }^{(4)}$.

Lyn C. Dirksen described a procedure of providing more natural appearance of buccal and labial contours of complete dentures. Outer surface of cured denture base should be carved and finished during waxing up to achieve ultimate esthetic results during stippling ${ }^{(1)}$. Sureh Nayar and Nicholas W. Craik had stated that gingival stippling loss was a characteristic feature of inflamed gingiva. Copying gingival texture and contours enhances natural appearance of flanges through unequal light reflection through the following techniques; (1) tooth brush technique, (2) offset bur technique and (3) blue wax technique ${ }^{(5)}$

For more enhancement of natural effect, gingival anatomy and alveolus are introduced by marked prominence related to alveolar eminences over the roots especially canines and gradually decreased in premolar and molar regions. Inflamed gingiva reflection should be reproduced by leaving more interdental wax and loss of stippling ${ }^{(5)}$.

Festooning is shaping the waxed base to simulate normal tissues in prosthesis. Waxing up should have standard and normal look which must also aid in physiologic retention and neuromuscular control of prosthesis. Increased contour of the labial flange is avoided to prevent loss of interior negative pressure ${ }^{(6)}$.

According to Organization of World Health, there were about more than one million smoker around the world. In the oral cavity, the prosthesis materials were situated in complex processing fluctuations resulting in damaging compounds which resulting in changing the physical and biological characteristics of the acrylic resin which is called biodegradation phenomena ${ }^{(7)}$.

Byproducts of cigarettes may be in the tobacco substances burnout. In case of smoking patients wearing dentures, prosthetic materials were situated in too many harmful compounds such as (CO), aldehydes, radioactive (Po), (NH3), (Ni), (Ar), tar and heavy metals. Those compounds reported marked change in color, alteration of microhardness and increase the surface roughness of dental resins ${ }^{(8)}$.

However there were not several studies explaining method of restorative materials exposure to burnout compounds in standard mode, they evaluated the effect of burn out byproducts without using same equipments, cigarettes count, smoke flow and time of the material exposure to the agent ${ }^{(9)}$. 
Studies conducted that significant color change of resins exposed to the burnout byproducts from 40 cigarettes. Advanced studies revealed the effect of burnout byproducts incorporated with staining solutions showing that the association of these byproducts could enhance the color change of prosthesis ${ }^{(10)}$.

When this burnout byproducts contact with the prosthetic material surfaces, esthetics is highly compromised as the prosthetic material of the individual due to smoking become obviously color changed due to impregnation by burnout byproducts ${ }^{(8)}$.

Color is one of the most required properties of artistic clinical point of view, preservation of the same color for the entire life may contribute to the longevity of the prosthesis so the denture base should be resistant to color changes ${ }^{(11)}$.

Stability of color change is the capacity of any prosthesis to preserve its initial color. With the constant fluctuations of micro flora, salivary secretions and persistence of staining food, the color change of the prosthesis may be noticed. Moreover, several national and international standards as ADA specification no. 12 demonstrated the importance of color stability of denture base which affect its longevity ${ }^{(12)}$.

Color matching is the most applicable factor in relation to esthetic success of complete denture. Prosthesis discolorations follow three reasons: (1) external causes due to food accumulations and which induce plaque formation and staining; (2) Supra or subsurface changes enhancing surface degeneration, infiltration and reaction of staining compounds with the prosthesis interface (adsorption); and (3) internal causes due to chemical and physical alterations inside restorative material. discolorations are caused by the diffusion of staining compounds such as cigarettes affecting aesthetic restorative materials ${ }^{(13)}$.

The prerequisite of shade and translucency of denture base should be preserved during laboratory workout and these bases should not be discolored inside oral cavity. Optical characteristics of the material in oral conditions resulting in color mismatch. Esthetic characteristics enhanced by color perception, since the light with the optical properties will affect the perception of the prosthesis color. Color change is the most important factor that affecting the visual perception ability ${ }^{(14)}$.

So, roughness is an important and critical character of the resin because of its relation to the tissues due to microorganisms' accumulation. Inside oral environment, the resin material should have less surface roughness and more lustrous surface for clinical acceptance and for more prosthetic serviceability, good appearance, and less food microorganism's accumulation. These microorganisms were protected from removal forces by cleaning actions resulting in enhanced denture stomatitis, discolorations, halitosis and clinical nonacceptance. So, roughness is of a highly clinical relation to dentures longevity and serviceability ${ }^{(15)}$.

Researches have contributed todietary ingredients as main cause in prosthetic material discolorations resulting in clinical disagreement, extra time and cost for prosthetic treatment. Researches on tealeaves, coffee beans as coloring compounds were few to correlate their effect on prosthetic polymers, however researchers have revealed that color instability of temporary prosthesis, soft liners, resin teeth materials, crown and fixed partial denture resins ${ }^{(16)}$.

Evaluation of color perception in dentistry is achieved by visual or instrumental methods. Visual perception has been introduced by clinician's ability to radiant energy stimulation. Instrumental color perception gives higher ability over visual one ${ }^{(17)}$.

Instrumental spectrophotometers and colorimeters have been introduced mainly in dentistry. Spectrophotometers measure the reflectance or transmittance factors of an object one wavelength at a time. Spectrophotometers were more accurate than colorimeter in detecting color change. Spectrophotometers assess the curve of reflectance every $10 \mathrm{~nm}$ or less because they contain monochromators and photodiodes ${ }^{(18)}$. 


\section{MATERIALS AND METHODS}

Total 180 maxillary complete dentures were designed and fabricated as sample size; 90 for each group divided into 30 dentures for each subgroup of staining condition (smoking, tea and coffee). After trial jaw relation record was transferred to suitable articulator, maxillary anterior teeth were set-up to confirm the principles of facial esthetics and phonetics using tooth brush technique of waxing up. Tooth brush technique of stippling by using the bristles of tooth brush before flasking through pressing against the surface of the waxed up denture. This results in production of many tiny indentations in the wax up. This is slightly flamed to make the indentations less prominent and forming minute dimples. Anatomic cross-linked acrylic posterior teeth were modified, arranged and balanced following the guide lines of lingualized concept of occlusion.

The selected dentures were randomly divided into two equal groups according to the technique of denture flasking into; group I as control group: conventional three pour flasking technique using plaster, group II as test group: silicone mask flasking technique using silicone putty and plaster combination.

For group I (control group), the waxed trial denture bases were flasked in a metallic dental flask with dental plaster using three pour technique. The base of the flask was painted with vaseline and filled with dental stone. The cast with the waxed up denture base was immersed into the plaster filled base at the center of the flask.

Once the base was flasked, a separating medium was applied and the second pour was done. The flask was left for 30-60 minutes undisturbed till the investing stone reach its final setting time then as third pour was poured.

In group II (test group) following same procedures of trial denture waxing up, a measuring spoon was used to take the required quantity of heavy condensation type of laboratory silicone and spread it on the palm of hand. Pressing the edge of the measuring spoon onto the mass once for each spoonful used.

Two strips of catalyst gel were applied for every spoon used which is $4 \mathrm{~cm}$. folding the mass over on itself and kneading it with finger tips for about 30 seconds until color is even without strips. Mix applied gingivally on the labial and palatal aspects of waxed up denture flange and usual flasking procedures performed as group I, as showed in figure (1).

For both groups, wax elimination was done by placing the flask in boiling water for 15 minutes. After 15 minutes, the flask was taken out of the water bath. The base and the body were carefully separated and the softened wax was flushed out using hot water stream. The powder and the liquid of the heat cured acrylic resin were mixed following the manufacturer's instructions and packed into the mold when the mix reached the dough stage. Pressure was applied on the flask after the first packing within 100- 150 Bar using calibrated compressor.

After the final closure of the flask, it was kept at room temperature for 30-60 minutes for bench curing, and then flask was heated in water bath following the curing cycle $\left(72^{\circ} \mathrm{C}\right.$ for $6.5 \mathrm{~h}$ and $92^{\circ} \mathrm{C}$ for $1.5 \mathrm{~h}$ ). The flask was cooled slowly and deflasking was done carefully to avoid cast fracture. Dentures were decasted, finished, polished and stored in tap water for 24 hours.

(E), color stability, was calculated for both groups immediately and after seven days of cigarette smoke exposure into specifically designed smoke chamber then $\Delta \mathrm{E}$, color stability change, was calculated. Custom made smoke chamber was made of insulating material that has a separable lid to close the chamber hermetically. Volume of smoke chamber was kept as minimum as possible. For entry of cigarette smoke; an inlet was designed at the center of the chamber lid to fit cigarette holder in which lit cigarette can be snugly fitted, opposed by chamber outlet opening of the same diameter. 


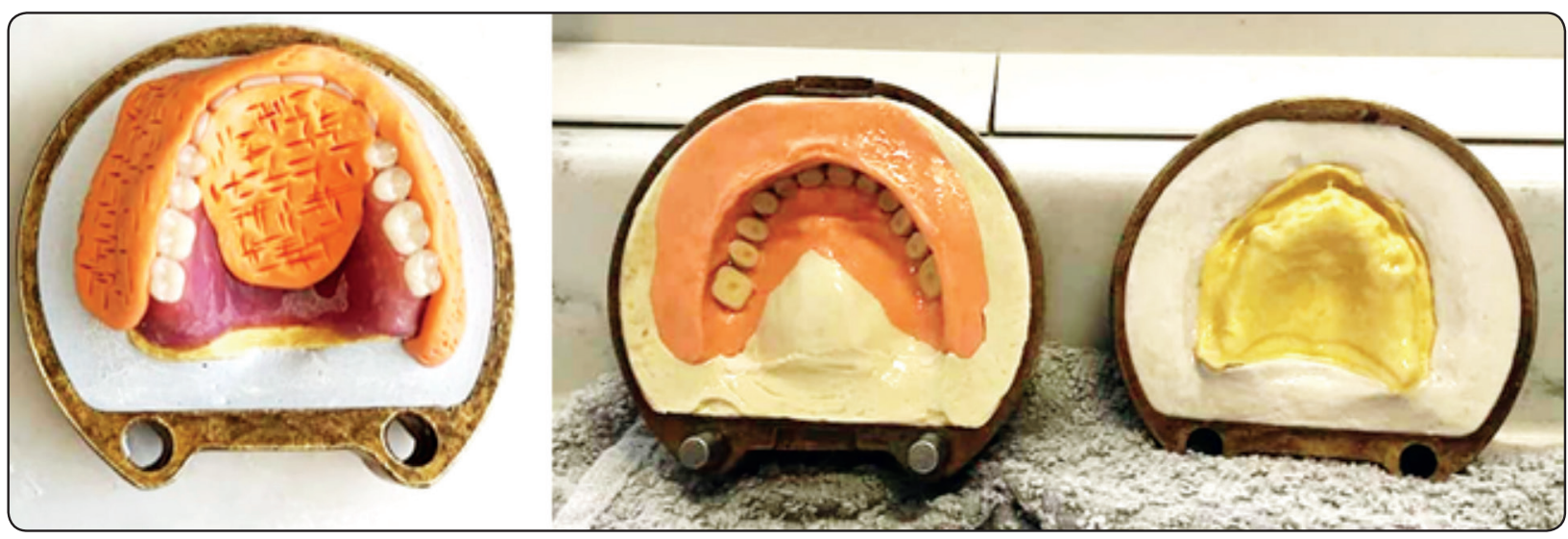

Fig. (1): Silicon Mask Flasking Technique

To mimic the process of smoking in-vivo, the smoke chamber was connected to a vacuum system, which was adjusted to maintain a steady flow rate of smoke. For active filling of cigarette smoke in smoke chamber, outlet was closed and vacuum system was switched on for 2 seconds, so as to mimic active inhalation of smoke in-vivo (puff duration). To maintain passive exhaust of smoke, the outlet was left open for 60 seconds before the next smoke cycle will be performed, so as to mimic passive exhalation of smoke in-vivo.

All 30 dentures of each subgroup (test group) was exposed to nicotine smoke of 6 cigarettes daily for seven days in a smoke chamber. For each cigarette, 9 puff cycles will be carried out. Each cycle will contain puff duration of 2 seconds and a puff frequency of 1 puff every 60 seconds.

Solutions of tea and coffee were prepared by adding $8 \mathrm{~g}$ of colorant to $400 \mathrm{ml}$ boiling distilled water. It was allowed to cool for $10 \mathrm{~min}$ and then filtered through a piece of gauze. After each cycle of exposure, dentures were washed for $1 \mathrm{~min}$. and then stored in distilled water at $37^{\circ} \mathrm{C}$.

The dentures' color were measured using a portable reflective spectrophotometer (X-Rite, model RM200QC, Neu-Isenburg, Germany). The aperture size was set to $4 \mathrm{~mm}$ and the specimens were exactly aligned with the device. A white background was selected and measurements were made according to the CIE $\mathrm{L}^{*} \mathrm{a} * \mathrm{~b} *$ color space relative to the CIE standard illuminant D65 where; $\mathrm{L}^{*}=$ lightness $(0-100), \mathrm{a}^{*}=$ (change the color of the axis red/green) and $b^{*}=$ (color variation axis yellow/ blue), as showed in figure (2).

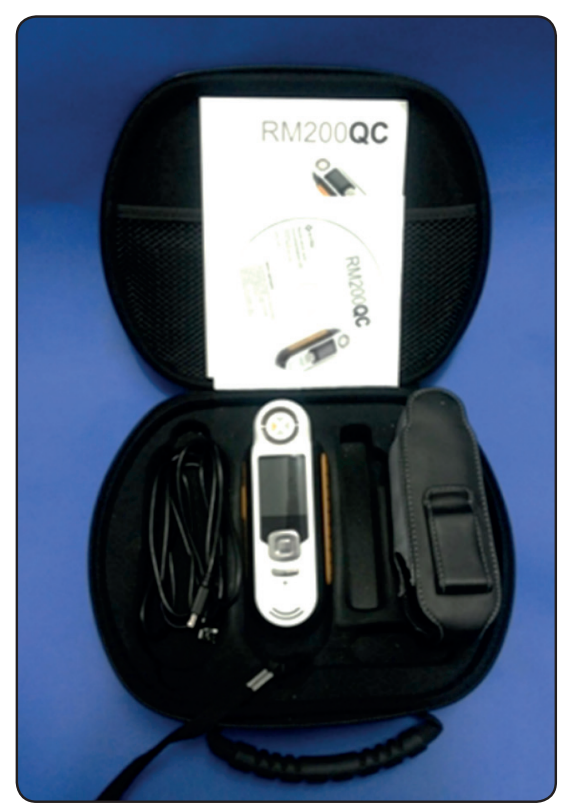

Fig. (2): Spectrophotometer 


\section{RESULTS}

The following formula was used to evaluate color changes $(\Delta \mathrm{E})$, color change, of the dentures

$$
\Delta E^{*}=\sqrt{\Delta L^{* 2}+\Delta a^{* 2}+\Delta b^{* 2}}
$$

Where; $\mathrm{L}^{*}=$ lightness $(0-100), \mathrm{a}^{*}=($ change the color of the axis red/green) and $b^{*}=$ (color variation axis yellow/blue)

Regarding smoking conditions, mean \pm standard deviation values of $(\Delta \mathrm{E})$ for group I (control group) and group II (study group) were $(3.97 \pm 0.304)$ and $(3.1 \pm 0.42)$ respectively as listed in table (1) and showed in figure (3). While for tea coloring agent, mean \pm standard deviation values of $(\Delta \mathrm{E})$ for group I (control group) and group II (study group) were $(3.6 \pm 1.1)$ and $(2.9 \pm 0.84)$ respectively as listed in table (1) and showed in figure (2). Finally, for coffee coloring agent, mean \pm standard deviation values of $(\Delta \mathrm{E})$ for group I (control group) and group II (study group) were $(3.5 \pm 0.4)$ and $(2.7 \pm 0.25)$ respectively

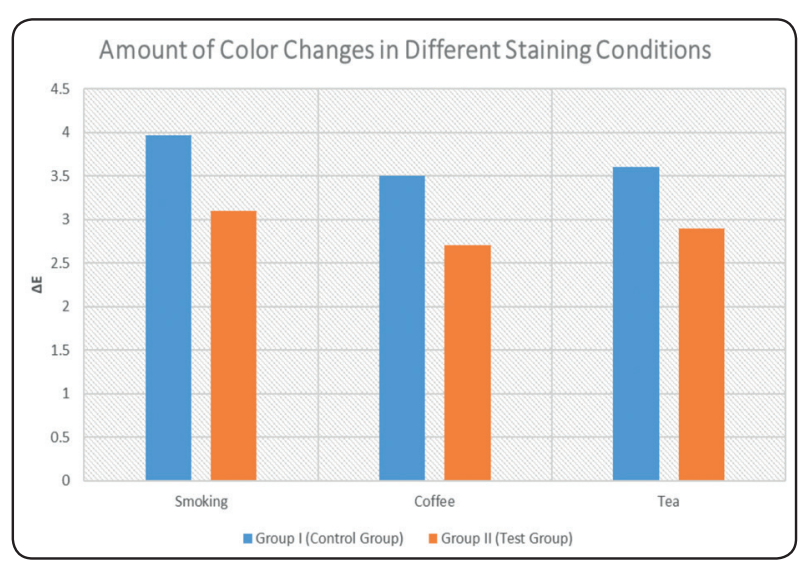

Fig. (3): Bar Chart of Color Changes $(\Delta E)$ for Comparison between Control Group (Group I) and Test Group (Group II) in Different Staining Conditions

as listed in table (1) and showed in figure (3).

At level of probability $\mathrm{P} \leq 0.05$, independent t-test was performed to evaluate the significance between both groups which revealed that there was significant difference between both groups as $\mathrm{P}$-value $<0.05$, as listed in table (1).

TABLE (1): Comparative values of Color Changes $(\Delta \mathrm{E})$ for Comparison between Control Group (Group I) and Test Group (Group II):

\begin{tabular}{|c|c|c|c|c|c|}
\hline & & $\mathrm{N}$ & $\begin{array}{c}\text { Group I } \\
\text { (Control Group) }\end{array}$ & $\begin{array}{c}\text { Group II } \\
\text { (Test Group) }\end{array}$ & P-value \\
\hline \multirow{3}{*}{$\begin{array}{l}\text { Color Changes } \\
\qquad(\Delta \mathrm{E}) \\
(\text { Mean } \pm \mathrm{SD})\end{array}$} & Smoking & 30 & $3.97 \pm 0.304$ & $3.1 \pm 0.42$ & $0.0001 * *$ \\
\hline & Coffee & 30 & $3.5 \pm 0.4$ & $2.7 \pm 0.25$ & $0.0001 * *$ \\
\hline & Tea & 30 & $3.6 \pm 1.1$ & $2.9 \pm 0.84$ & $0.0075 * *$ \\
\hline
\end{tabular}

N; Count, M; Mean, SD; Standard Deviation, P; Probability Level

**significant Difference 


\section{DISCUSSION}

CIE-lab scale, the most reliable scale, was used in this study as (L) reveals luminescence and starts from 0 (black) to 100 (white); also greenred axis represented by (a), while blue-yellow axis represented by (b). Accordingly, the equation was used to calculate the difference between two points distance on this scale which reveals hypotenuse in right angled triangle in 3D space ${ }^{(16)}$.

Acrylic prosthesis may absorb pigments dissolved in staining conditions may be carried into the resin body causing color changes of the prosthetic base. This absorption resulted from volatilizing some of its monomers or low molecular weight compounds resulting in the surface irregularities created when the temperature difference of resin body is above $100.8^{\circ} \mathrm{C}$ in addition to hydrophilic nature of polymethylmethacrylate ${ }^{(18)}$.

Tea leaves were processed by drying and fragmentation, which may lead to a great pigmentation accumulation of coloring agents in its solution which was similar to that tea has a higher color change ability than coffee. On the other hand, Imirzalioglu et al, 2010 found that pigmentation by tea had insignificance of difference. Future study will be needed on the kind of tea and leaf processing used as coloring agents ${ }^{(19)}$.

Several studies demonstrated that color stability was greatly affected by porosity and roughness. However our results were accepted with others found in the literature, staining ability of beverages depend upon frequency and amount of intake. Thus, any given beverages has not significant effect on the esthetics of a denture base materials in the same manner as in this study ${ }^{(16)}$.

During smoking there is release of nicotine, ammonia, nickel, arsenic, carbon monoxide, carbon dioxide, tar and heavy metals which are active component of cigarette. Temperature increased within the oral cavity by smoking. During exposure to smoke, all the dentures were also placed in the artificial saliva to simulate intra oral conditions. It was found that those dentures which were exposed to cigarette smoke showed surface roughness due to accumulation of tobacco products ${ }^{(9)}$.

\section{CONCLUSION}

With the limitations of this study regarding color stability change $(\Delta \mathrm{E})$, depending on flasking technique; the conventional three pour flasking technique showed a higher amount of color change (lower color stability) than the silicone mask flasking technique regarding smoking, tea and coffee coloring agents.

\section{REFERENCE}

1. Keith J. Ferro, DMD Committee Chairman, Committee of the Glossary of Prosthodontic Terms, Edition Nine Academy of Prosthodontics, Glossary of Prosthodontic Terms, Edition One, J Prosthet Dent 1956;1-34.

2. Ahrari F, Heravi F, Rashed R, Zarrabi MJ, Setayesh Y.; Which factors affect dental esthetics and smile attractiveness in orthodontically treated patients, J Dent (Tehran) 2015; 12:491-503.

3. Zarb GA, Bolender CL, Carlsson GE, Boucher CO. Boucher's Prosthodontic; Treatment for edentulous Patients. $13^{\text {th }}$ ed. St. Louis: Mosby; 2012.

4. Kreyer R. Gingival characterization. Heal Med Coll 2007: $32: 46$.

5. Anand, V., \& Manoharan, P. S.; The dynamics of pink and white esthetics in complete denture. $20196,78-82$.

6. Pattanaik S, Pattanaik B.; Internal characterization of denture base by using acrylic stains and tissue paper. J Indian Prosthodont Soc 2011; 11:202-4.

7. Ayaz EA, Altintas SH, Turgut S.; Effects of cigarette smoke and denture cleaners on the surface roughness and color stability of different denture teeth. J Prosthet Dent. 2014; 112:241-48.

8. Alandia-román, C. C., Cruvinel, D. R., Sousa, A. B. S., \& Panzeri, H.; Effect of cigarette smoke on color stability and surface roughness of dental composites Effect of cigarette smoke on color stability and surface roughness of dental composites. Journal of Dentistry, 41(March 2019), e73-e79. 
9. Mathias P, Rossi TA, Cavalcanti AN, Lima MJ, Fontes CM, Nogueira-FilhoGda R.; Cigarette smoke combined with staining beverages decreases luminosity and increases pigmentation in composite resin restorations. Compend Contin Educ Dent. 2011; 32:66-70.

10. Topcu, F. T., Sahinkesen, G., Yamanel, K., Erdemir, U., Oktay, E. A., \& Ersahan, S.; Influence of Different Drinks on the Color Stability of Dental Resin Composites. European Journal of Dentistry, 2009; 03(01), 50-56.

11. Sari, N. M. G. A. W., Fardaniah, S., \& Masulili, C.; Color changing in denture base polyamide 12 and polyamide microcrystalline after polishing in laboratory and dental clinic. Journal of Physics: Conference Series, 2017, 884(1).

12. Sagsoz, N. P., Yanıkoglu, N., Ulu, H., \& Bayındır, F.; Color Changes of Polyamid and Polymetyhl Methacrylate Denture Base Materials. Open Journal of Stomatology, 2014, 04(10), 489-496.

13. Goiato, M. C., Micheline, D., \& Moreno, A.; Effect of different solutions on color stability of acrylic resin based dentures. 2014, 28, 1-7.
14. Ideiaslab-Prótese Flexível PPFlex. [Internet]. Rio de Janeiro [cited 2009 Apr 15]. http://www.ideiaslab.com.br

15. Jain V, Platt J A, Moore K, Spohr A M and Borges G A; Color stability, gloss, and surface roughness of indirect composite resins J. Oral Sci. 2013, 55 9-15.

16. Vafaee, F., Tavakolizadeh, S., Kadkhodazadeh, M., \& Khoshhal, M.; Evaluation of the Effect of 2 Flask Investment Materials on Color Stability of 5 Brands of Denture Teeth. 2014, 3(4), 144-150.

17. Hatim N A and Al-Tahho O Z; Comparative evaluation of color change between two types of acrylic resin and flexible resin after thermo cycling. an in vitro study. J. Indian Prosthodont. 2013 Soc. 13 327-337.

18. Waldemarin, R. F. A., Terra, P. C., Pinto, L. R., Faot, F., \& Camacho, G. B.; Color change in acrylic resin processed in three ways after immersion in water, cola, coffee, mate and wine, Acta Odontol. Latinoam. Vol. 26 N N $^{\circ}$, 2013, 138-143. 